\title{
Wavelet Analysis of EEG Signals in Epilepsy Patients
}

\author{
Tatyana V. Yakovleva*[0000-0003-3238-2317], Ilya E. Kutepov ${ }^{[0000-0002-1003-4496]}$, \\ Anton V. Krysko ${ }^{[0000-0002-9389-5602]}$, Nikolay P. Erofeev ${ }^{[0000-0002-2466-1159]}$, \\ Tatyana Yu. Yaroshenko ${ }^{[0000-0002-4914-764]}$, Olga A. Saltykova ${ }^{[0000-0002-3880-6662]}$, \\ Anastasiya V. Kirichenko, Maxim V. Zhigalov ${ }^{[0000-0002-0642-7211]}$, \\ Irina V. Papkova ${ }^{[0000-0003-4062-1437]}$, Vadim A. Krysko ${ }^{[0000-0002-4914-764]}$ \\ Yuri Gagarin State Technical University of Saratov, \\ Politehnicheskaya, 77 Saratov, Russian Federation \\ *yan-tan1987@mail.ru \\ Nikolay M. Yakovlev ${ }^{[0000-0003-4127-035 X]}$, Antonina Yu. Karas ${ }^{\dagger}$ \\ Medical center of neurology, diagnosis and treatment of epilepsy "Epineiro", \\ Bolshaya Sadovaya, 151/8, Saratov, Russian Federation \\ †antoninakaras@yandex.ru
}

\begin{abstract}
The paper presents studies of electroencephalogram (EEG) signals of epilepsy patients based on wavelet transform and calculation of the total signal energy. The object of the study was a patient aged 17-22 years diagnosed with focal (structural) epilepsy, mesial sclerosis on the left and focal cortical dysplasia of the left temporal lobe, and a control group. The results were compared in the form of 3D Morle wavelets and topographic images. Topographic images of the head surface are obtained on the basis of the integral energy of brain signals. The study showed that wavelet analysis of EEG signals can be a useful tool in the study of EEG signals both in epilepsy patients and in comparison with the control group. It is assumed that such analysis will be useful for early detection of neurological changes and decision-making by doctors for further treatment.
\end{abstract}

Index Terms-EEG signal, epilepsy, peak wave, sharp wave, wavelet analysis, total energy, visualization, nonlinear dynamics, decision-making

\section{INTRODUCTION}

Modern times are characterized by huge flows of information that a person needs to perceive, understand and apply to solve current problems. This situation of making the right decisions is associated with great emotional and physical stress. Therefore, studies of human brain activity are becoming more relevant. When studying the capabilities and features of the human brain, it is necessary to conduct studies of both the brain activity of a healthy person and people with various paroxysmal disorders. Paroxysmal States are observed in various neurological diseases, the most dramatic among them is epilepsy. This disease affects more than $1 \%$ of the world's population and the dynamics of its spread only in developed countries - cases per year. Moreover, the disease can be both hereditary and acquired, the so-called post traumatic epilepsy (the etiology of epilepsy is diverse, it can be hereditary and acquired). Diagnosis of epilepsy is based on the analysis of electroencephalogram (EEG) signals, which are actually chaotic time series. Methods of analysis and classification of EEG signals are based on the fact that the processing of information in the brain is reflected in the EEG as dynamic changes in electrical activity in time, frequency and space. Such problems can be solved with the help of such a method of nonlinear dynamics as wavelet analysis.

Wavelet analysis as a "mathematical microscope" is a universal tool for analyzing time series of any nature [1]. In the review [2], the authors emphasize that the wavelet transform as a mathematical microscope allows the analysis of EEG signals at different scales [3]. Such well-known wavelets as Daubechies, Mexican Hat, DWT are used to study epileptic EEG signals [4]-[6]. In addition, a number of works use wavelets constructed by the authors. In [7] the wavelet-ApEn (wavelet approximation entropy) method is used to detect epilepsy in EEG signals. Studied 5 groups of EEG signals (healthy with open eyes, healthy with closed eyes, interictal and ictal activity). When comparing some groups, the reliability of more than $99.98 \%$ was achieved for the EEG and all its sub-bands. In [8], using the author's wavelet and the algorithm for constructing Granger causeeffect relationships, the EEG signal was studied to objectively check the lateralization of zones affected by temporal lobe epilepsy The result of the wavelet analysis confirmed by Granger cause-effect relationships showed that high-frequency bursts on EEG signals are initiated and propagated in the local area of the brain, then propagated to adjacent areas. In [9] using the signal energy on the basis of the Morle wavelet and entropy identifies differences between predictably and interictal periods in EEG signals. This is the first step in predicting seizures in patients with mesial temporal lobe epilepsy. In [10]-[12] EEG signals are decomposed by frequency using discrete wavelet transform (DWT). This method is used to extract signs of epileptiform activity from 
significant drawback: the lack of localization of frequencies

EEG signals.

Effective features of signals are determined by the relative energy and amplitude, coefficient of variation and fluctuation. In the article [13] the authors proposed a method based on tunable wavelet transform coefficient for a utomatic diagnosis of epilepsy. This method of detecting pathological activity can speed up the diagnosis of epileptic seizures. The packet wavelet transform (WPT) was used in [14] to create a clinically useful automated seizure detection system for EEG recordings. In [15], the packet wavelet transform is used to analyze EEG data and to obtain characteristics of the time and frequency domains and statistics of pattern coincidence regularity (PMRS). These data are used to quantify the complexity of the EEG time series. The resulting EEG feature vectors are recognized by the weighted elm extreme learning machine.

Another application of wavelet transform in the study of EEG signals is the purification of $\mathrm{s}$ ignals $\mathrm{f}$ rom $\mathrm{n}$ oise and its decomposition into different frequency sub-bands. The wavelet transform [16] is used to decompose EEG signals into several frequency sub-bands. For all nodes, on the basis of the Kruskal-Wallis criterion, signs characterizing epilepsy are selected. In [17] epileptiform activity on the EEG was decomposed into frequency sub-bands using the Daubechies wavelet. The frequencies of the selected sub-bands were used as input to the trained neural network for classification of EEG signals. The studies were conducted on the basis of a sample of 5 healthy and 5 patients with temporal lobe epilepsy. In [18] epileptiform activity on the EEG was pre-processed using the threshold wavelet method to remove artifacts. This allows you to identify the characteristic features in the time domain, frequency domain, time-frequency domain. These characteristics are distinguished from five frequency sub-bands depending on clinical goals. Using DWT [19], artifacts from pathological EEG signals are removed and signs characteristic of epilepsy are distinguished. The results showed that wavelet analysis was an effective method for the automatic diagnosis of epilepsy by studying EEG signals. This review shows that the wavelet transform is usually used in two ways: directly for the study of the EEG signal and as a tool for cleaning the signal from noise, splitting into frequency ranges and as input parameters of a self-learning neural network.

In this paper, we use wavelet to determine the total energy of the EEG signal. This will reveal abnormal brain activity. Morle wavelet is used for this task, which has proven itself in the study of signals of different nature: historical [20], mechanical [21], [22]. Frequency analysis of EEG signals will also be carried out.

\section{WAVELET TRANSFORMATION}

One of the main and universal characteristics of oscillations in various systems is the power spectrum of the oscillatory process. Typically, a fast Fourier transform is used to estimate it from the available counts of the observed quantity in time. The resulting spectrum-information about the energy distribution of the oscillatory process-has one in time, which is especially important in the study of signals with a time-varying frequency spectrum. These signals include signals obtained in the study of the brain using electroencephalogram (EEG) and magnetic resonance imaging (MRI). To obtain the time-frequency spectrum in the mid80s of the last century Grossman and Morle [23] proposed a mathematical apparatus called "wavelets" (from the English. wavelet, which literally means "small wave"). The emergence of the theory of wavelets is considered one of the most important events in mathematics in recent decades, since this theory, immediately after its appearance, was perceived as a tool for applied research in almost all natural Sciences and many fields of technology. Currently, a family of analyzers called wavelets is widely used in the problems of pattern recognition, radiographs of human internal organs, satellite images of clouds or the surface of the planet, to study the properties of turbulent fields, etc., whereas in the nonlinear dynamics of the theory of beams, plates and shells, they are practically not used. By analogy with the Fourier transform, the wavelet transform of a signal consists in its expansion over a certain basis constructed from a solitonlike function (wavelet) with certain properties by means of scale transformations and transfers. Each of the basis functions characterizes both frequency and localization in time. Thus, the wavelet transform provides a two-dimensional sweep of the signal, with the frequency and time considered as independent variables. This makes it possible to analyze the properties of the signal simultaneously in time and in private space. The continuous wavelet transform of signal $x(t)$, defined in the time domain $t \in(-\infty ;+\infty)$, has the following form:

$$
W\left(\omega, t_{0}\right)=\int_{-\infty}^{+\infty} x(t) \Psi_{\omega, t_{0}}^{*}(t) d t
$$

where $\Psi_{\omega, t_{0}}(t)$ - wavelet function, which is derived from the "mother" wavelet $\Psi_{0}(t)$ :

$$
\Psi_{\omega, t_{0}}(t)=\frac{1}{\sqrt{\omega}} \Psi_{0}\left(\frac{t-t_{0}}{\omega}\right) .
$$

Value $\omega$ enters the window width of the wavelet, $t_{0}-$ shifts the wavelet function along the time axis, $*$ defines complex conjugation. Since the wavelet transform is the scalar product of the analyzing wavelet and the analyzed signal, the coefficients $W(\omega, t)$ contain combined information about the wavelet and the signal (as well as the Fourier transform coefficients, which contain information about the signal and the sine wave). In this paper, we will use some types of wavelets to analyze EEG signals and show that each of the considered wavelets has characteristic features in time and frequency space. Many researchers call wavelet analysis a "mathematical microscope" because the method retains good resolution at different scales. The shift parameter $t_{0}$ captures the focus point "mathematical wavelet microscope" (this name reflects the remarkable property of the method to maintain a good resolution at different scales), the scale factor $s-$ is 
the magnification, and finally, the "optical quality" of the microscope are determined by the choice of reference wavelet $\Psi$. The most frequently used basis is constructed on the basis of a well-localized in $f$ and $t$ Morlet wavelet in space [23], which is a plane wave modulated by a unit-width Gaussian. The following is the expression of the corresponding wavelet function and its Fourier transform:

$$
\Psi_{m}(t)=e^{\left(i f_{0} t\right)} e^{\left(-\frac{|t|^{2}}{2}\right)}, \hat{\Psi}_{m}(f)=H(f) e^{-\frac{\left(f-f_{0}\right)^{2}}{2}},
$$

where $H(f)$ - is Heaviside function. Note that the angular selectivity $k_{0}$ of the basis increases with increasing, but the spatial selectivity deteriorates. In this paper we will use a variant of graphic images of the wavelet transform in the form of a three-dimensional surface in a Cartesian rectangular coordinate system, where time, frequencies and modules of the wavelet coefficients are plotted along the axes, respectively. Morlet wavelet was chosen as the mother wavelet. The value of one of the wavelet parameters $f_{0}=2 \pi$ is chosen to provide the relation $\omega \approx 1 / \hat{\omega}$, where $\omega$ is the time scale of the wavelet transform, $\hat{\omega}-$ the Fourier transform frequency. The paper considers the value of

$$
\tilde{E}=\int_{0}^{S_{\max }} \int_{t_{1}}^{t_{2}}\left|W\left(\omega, t_{0}\right)\right| d t d S,
$$

which represents the total energy of the signal. Here $t_{1}, t_{2}$ is the initial end time of the study, respectively.

\section{Electrode Placement System}

EEG is one of the methods that allow you to study the human brain. The basis of this method is the registration of electrical impulses from the brain or some of its individual areas using an encephalograph. The electrodes pick up and transmit microcurrents in EEG amplitude change at each time check. Modern EEG systems use a variety of electrodes (16-256), which determine the voltage at the appropriate points of the human scalp.

The international Association of electroencephalography and clinical neurophysiology has developed an EEG diversion system that is common worldwide and mandatory for all researchers. The developed system of location of electrodes on the scalp allows to correlate the area of the electrode location with the underlying structure of the brain in this area. This system is called the "international 10-20 \% system". The graphic scheme is shown in Fig. 1. Lead points are indicated by large Latin letters corresponding to the initial letters of the zone name. Odd numbers mark the points of the left hemisphere. Electrodes located on the middle line are indicated with the index $\mathrm{z}$ : Fz, Cz, Pz. Ear electrodes are denoted by the letter A-A1, A2.

\section{The PARAmeters OF THE STUdy}

Wavelet analysis was used in the study of the EEG of a patient who was treated for 5 years - from 17 to 21 years. Diagnosis-focal (structural) epilepsy, mesial sclerosis on the left and focal cortical dysplasia of the left temporal lobe.

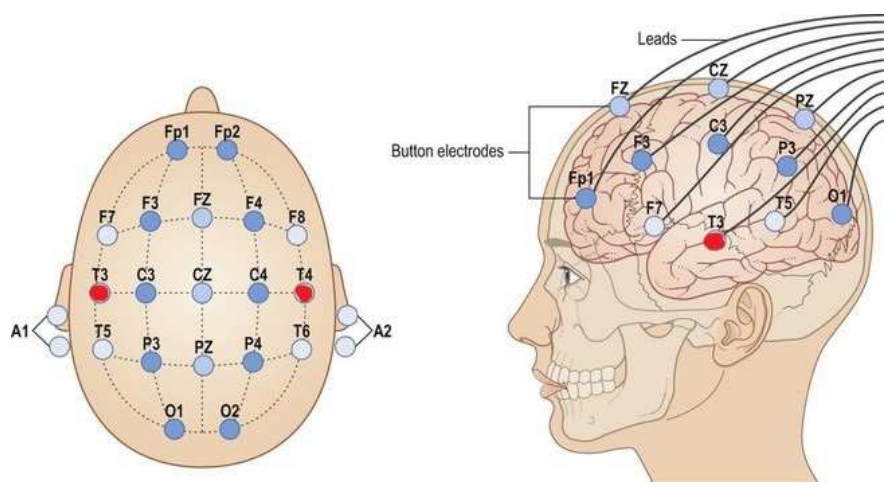

Fig. 1. The system of electrodes placement "10-20".

The EEG recording of a patient with epilepsy was performed in the medical center neurology diagnosis and treatment of epilepsy "Epineiro" (Saratov). During the examination, the EEG analysis was used with the electrode arrangement scheme shown in Fig. 1. The EEG recording was performed on 21 channel: O2, O1, P4, P3, C4, C3, F4, F3, Fp2, Fp1, T6, T5, T4,T3, F8 ,F7, Pz, Cz, Fz, A2, A1. Cleaning of artifacts was performed by a neurophysiologist. The study was conducted for all 21 channels. For rice. 1 the points for which the results are given in this paper are highlighted in red. The research results are given for three years: 2014, 2017, 2019.

\section{RESUlts}

The paper analyzed EEG signals containing pathological changes: "sharp wave" and "peak-wave" (according to the international classification of EEG disorders [24]). These pathological changes were compared with the signal without pathologies. The results are given in Fig. 2, 3. The first column provides explanatory information. The second column shows the wavelet surface $W(\omega, t)$ for the point T3. The third column shows the wavelet surface $W(\omega, t)$ for the point T4. The fourth column shows topographic images visualizing brain activity determined by channel 21. Fig. 2 shows the results for the peak-wave phenomenon. A peak-wave is a complex with a large amplitude resulting from the combination of a peak with a slow wave.

3D wavelets in 2014 for channels T3 and T4 are qualitatively different, the peak power frequency $\omega=8$ in the area channel T3 are present in all time interval, while the channel of $\mathrm{T} 4$ at the time $t=5 \mathrm{~s}$ they shift to a higher range. The frequency power (the value of the wavelet coefficients $W(\omega, t))$ of the E3 channel is greater. This is reflected in the topological image of the energy integral - in the area of the left ear there is a maximum (red). The results of 2017 have significant differences from 2014. The maximum of the wavelet coefficients $W(\omega, t)$ of the T4 channel is twice as large as that of the T3 channel, i.e. there is mirroring. On 3D wavelets at the time $t=5 \mathrm{~s}$ is a moment of sharp increase in frequencies of both channels. However, there is a qualitative difference in these "bursts", i.e. there is an asymmetry in the energy distribution. Asymmetric arrangement of energy along 
T3-A
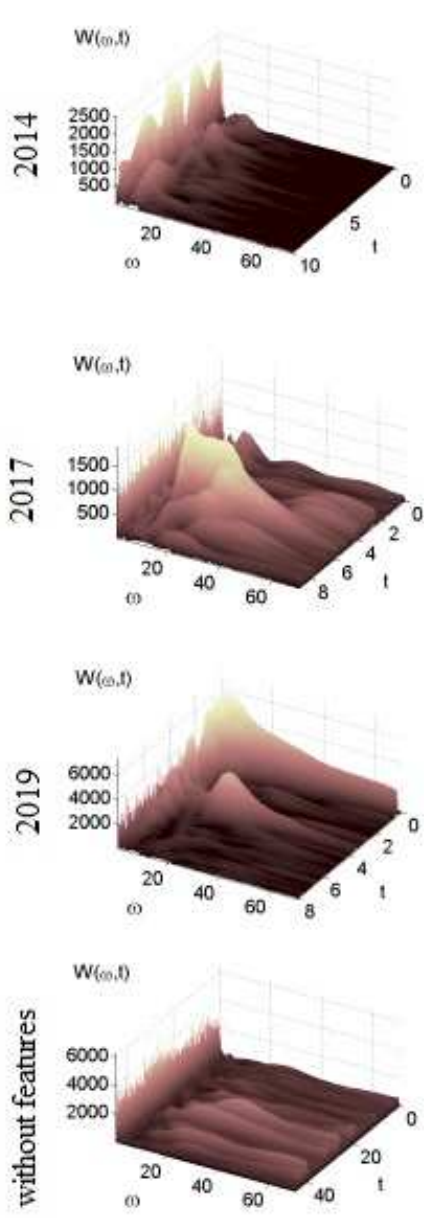

T4-A
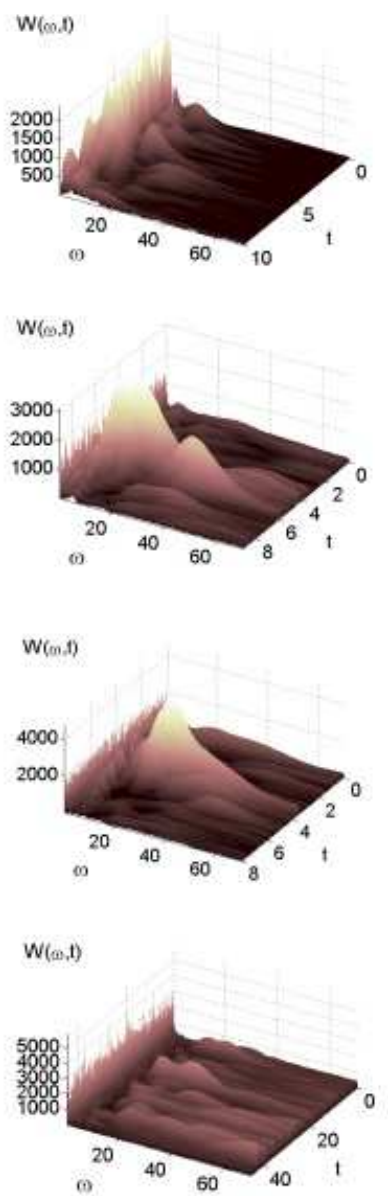

integral energy of channels
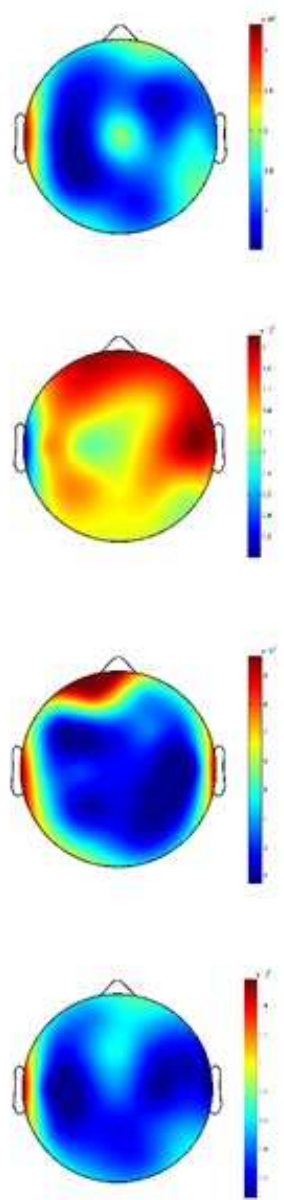

Fig. 2. Peak-wave.

the channel is also present on the topology of the energy integral. It should be noted that in the frontal part of the head there is a zone of maximum energy values with a maximum near the point Fp1. This zone is localized in 2019, where the topology of the energy integral has three zones of maximachannels Fp1, T3 and T4. On 3D wavelets both channels have a symmetrical "surge of power" frequencies at $t=3 \mathrm{~s}$. Note that again there is a change of the maxima of the wavelet coefficients - the channel T3 values are 1.5 times greater than the T4 channel. Comparison with the results without features (fourth row of the Fig. 2) shows that the closest results with 2014.

In Fig. 3 shows the results for the sharp wave phenomenon. This phenomenon has a peak shape with a period of 80-200 ms. Outwardly, it resembles a peak and differs from it only in the length of time. Its amplitude can reach the same values as the amplitude of the peaks. Sharp waves and peaks are often combined with slow waves, forming a stereotypical complex. The sharp waves phenomenon is highly specific to epilepsy.
In 2014, the maximum of wavelet coefficients $W(\omega, t)$ is on T3 channel, but the total energy is greater on T4, which is reflected in the topology of the energy integral. In contrast to the results of the peak-wave (see Fig. 2) there is a weak symmetry on the topology of the energy integral-channels T3 and T4 have maximum, although different in magnitude. 3D wavelets differ significantly from each other. So on T4 channel the main energy is concentrated at higher frequencies $(\omega>$ 20). In 2017, 3D wavelets are qualitatively similar, both have "bursts" of power at low frequencies $(\omega \approx 5)$. However, on T4 channel, the total amount of energy is greater. The topology of the energy integral indicates the presence of three zones of maximum value: T3, T4, Fp1+Fz. In 2019, 3D wavelets of T3 and T4 channels differ significantly. This is due to a maximum at Fp1, which is visible on the energy integral topology, where just as for the peak wave in 2019, three maximums are present. Comparison with results means quality match 2017 (especially in topology) from the results of the case without features. 
T3-A
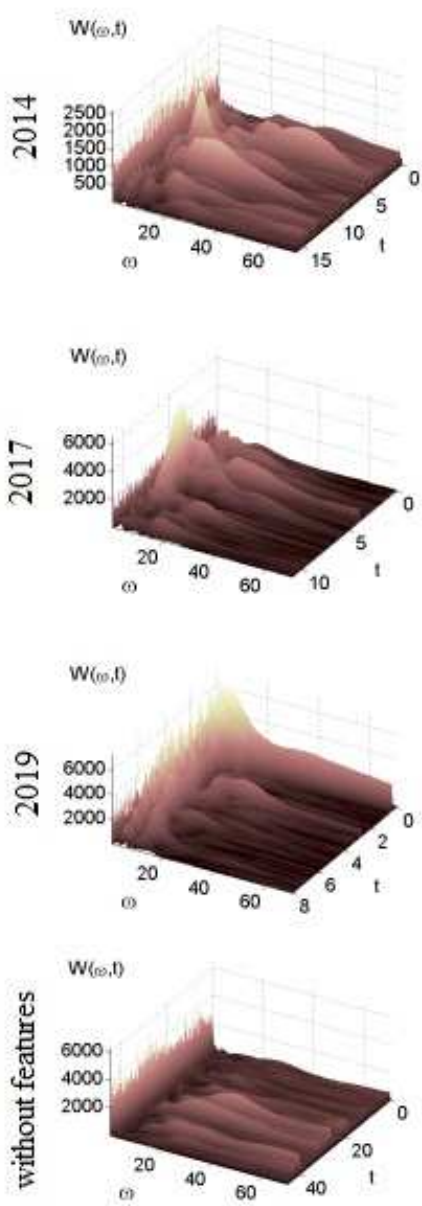

T4-A
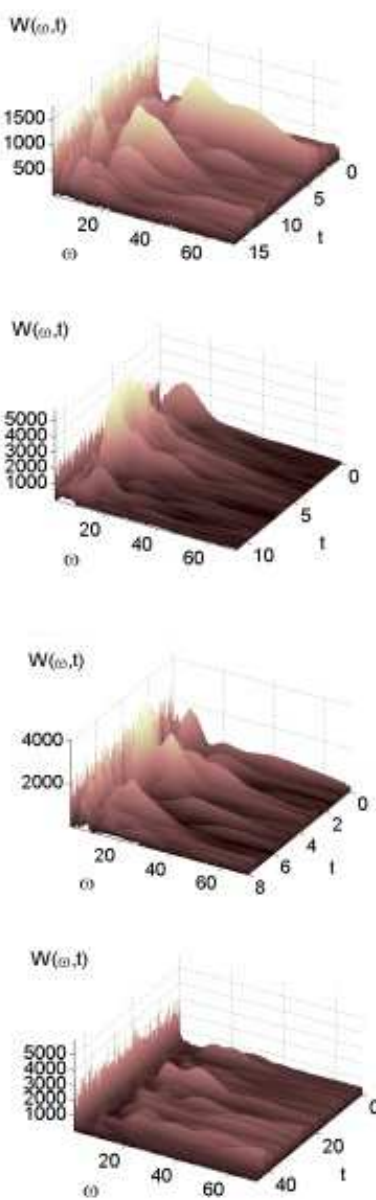

Fig. 3. Sharp wave. integral energy of channels
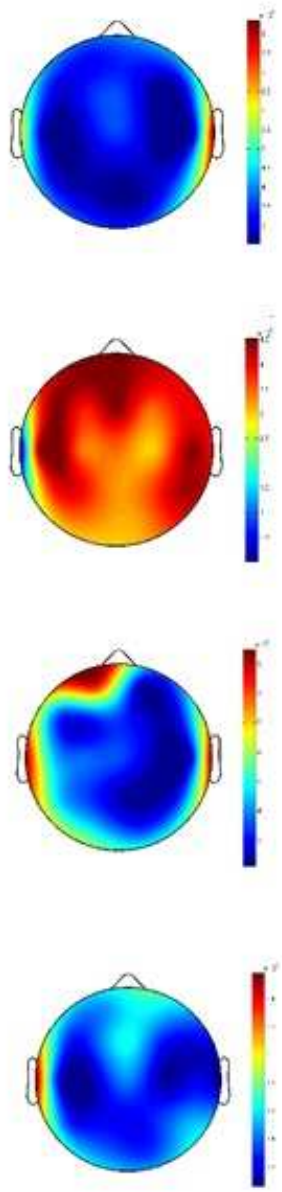

\section{CONCLUSION}

The aim of this work was to investigate the possibility of wavelet analysis and energy integral to detect abnormal brain activity. The 3D surfaces and topographic images made it possible to localize the brain areas in which pathological changes in the form of energy maxima are observed. The results are consistent with the medical history. In 2014, the frequency of attacks was 1 every six months. By 2017, there was a significant deterioration, and the frequency of seizures became in 20171 time per month, and in 2018 - 3 times per month. Such dynamics in the development of the disease is in good agreement with the graphs of energy distribution. Treatment was adjusted and the frequency of attacks in 2019 is 1 time in 1.5 months. The energy distribution topology for 2019 has become closer to 2014. The proposed concept of the study of EEG signals based on energy, in our opinion, will predict the course of the disease and will develop a decisionmaking system for the treatment of epilepsy.

\section{ACKNOWLEDGMENTS}

This work was supported by the Ministry of science and education of the Russian Federation No. 3.861.2017/HR.

\section{REFERENCES}

[1] N. Astafieva, "Wavelet analysis: fundamentals of theory and application examples," UFN, vol. 166, no. 11, pp. 1145-1170, 1996.

[2] O. Faust, U. R. Acharya, H. Adeli, and A. Adeli, "Wavelet-based eeg processing for computer-aided seizure detection and epilepsy diagnosis," Seizure, vol. 26, pp. 56-64, 2015.

[3] Y. Meyer, Wavelets and applications. Masson Paris, 1992, vol. 31.

[4] C. Avanzo, V. Tarantino, P. Bisiacchi, and G. Sparacino, "A wavelet methodology for eeg time-frequency analysis in a time discrimination task," International Journal of Bioelectromagnetism, vol. 11, no. 4, pp. 185-188, 2009.

[5] I. Omerhodzic, S. Avdakovic, A. Nuhanovic, and K. Dizdarevic, "Energy distribution of eeg signals: Eeg signal wavelet-neural network classifier," Engineering and Technology, no. 61, 2010.

[6] D. Chen, S. Wan, J. Xiang, and F. S. Bao, "A high-performance seizure detection algorithm based on discrete wavelet transform (dwt) and eeg," PLOS ONE, vol. 12, no. 3, 2017. 
[16] U. R. Acharya, F. Molinari, S. V. Sree, S. Chattopadhyay, K.-H. Ng, and J. S. Suri, "Automated diagnosis of epileptic eeg using entropies," Biomedical Signal Processing and Control, vol. 7, no. 4, pp. 401-408, 2012.

entropy method for epileptic activity detection from eeg and its subbands," Journal of Biomedical Science and Engineering, vol. 3, no. 12, p. 1182, 2010.

[8] T. Han, Z. Xu, J. Du, Q. Zhou, T. Yu, C. Liu, and Y. Wang, "Ictal high-frequency oscillation for lateralizing patients with suspected bitemporal epilepsy using wavelet transform and granger causality analysis," Frontiers in Neuroinformatics, vol. 13, 2019.

[9] K. Gadhoumi, J.-M. Lina, and J. Gotman, "Discriminating preictal and interictal states in patients with temporal lobe epilepsy using wavelet analysis of intracerebral eeg," Clinical neurophysiology, vol. 123, no. 10, pp. 1906-1916, 2012.

[10] Y. Liu, W. Zhou, Q. Yuan, and S. Chen, "Automatic seizure detection using wavelet transform and svm in long-term intracranial eeg," IEEE transactions on neural systems and rehabilitation engineering, vol. 20, no. 6 , pp. 749-755, 2012.

[11] H. Ocak, "Automatic detection of epileptic seizures in eeg using discrete wavelet transform and approximate entropy," Expert Systems with Applications, vol. 36, no. 2, pp. 2027-2036, 2009.

[12] Y. Li, H.-1. Wei, and S. A. Billings, "Identification of time-varying systems using multi-wavelet basis functions," IEEE Transactions on Control Systems Technology, vol. 19, no. 3, pp. 656-663, 2010.

[13] A. R. Hassan, S. Siuly, and Y. Zhang, "Epileptic seizure detection in eeg signals using tunable-q factor wavelet transform and bootstrap aggregating," Computer methods and programs in biomedicine, vol. 137, pp. 247-259, 2016

[14] D.-S. Shiau, J. Halford, K. Kelly, R. Kern, M. Inman, J.-H. Chien, P. Pardalos, M. Yang, and J. C. Sackellares, "Signal regularitybased automated seizure detection system for scalp eeg monitoring," Cybernetics and systems analysis, vol. 46, no. 6, pp. 922-935, 2010.

[15] Q. Yuan, W. Zhou, L. Zhang, F. Zhang, F. Xu, Y. Leng, D. Wei, and M. Chen, "Epileptic seizure detection based on imbalanced classification and wavelet packet transform," Seizure, vol. 50, pp. 99-108, 2017.

[17] A. Subasi, "Eeg signal classification using wavelet feature extraction and a mixture of expert model," Expert Systems with Applications, vol. 32, no. 4, pp. 1084-1093, 2007

[18] L. Wang, W. Xue, Y. Li, M. Luo, J. Huang, W. Cui, and C. Huang, "Automatic epileptic seizure detection in eeg signals using multi-domain feature extraction and nonlinear analysis," Entropy, vol. 19, no. 6, p. 222, 2017.

[19] O. Faust, U. R. Acharya, H. Adeli, and A. Adeli, "Wavelet-based eeg processing for computer-aided seizure detection and epilepsy diagnosis," Seizure, vol. 26, pp. 56-64, 2015.

[20] T. Y. Yaroshenko, D. V. Krysko, V. Dobriyan, M. V. Zhigalov, H. Vos, P. Vandenabeele, and V. A. Krysko, "Wavelet modeling and prediction of the stability of states: the roman empire and the european union," Communications in Nonlinear Science and Numerical Simulation, vol. 26, no. 1-3, pp. 265-275, 2015.

[21] J. Awrejcewicz, O. A. Saltykova, M. V. Zhigalov, and P. Hagedorn, "Analysis of non-linear vibrations of single-layered euler-bernoulli beams using wavelets," International Journal of Aerospace and Lightweight Structures (IJALS), vol. 1, no. 2, pp. 203-219, 2011.

[22] J. Awrejcewicz, I. Papkova, E. Krylova, and V. Krysko, "Wavelet-based analysis of the regular and chaotic dynamics of rectangular flexible plates subjected to shear-harmonic loading," Shock and vibration, vol. 19, no. 5, pp. 979-994, 2012.

[23] A. Grossmann and J. Morlet, "Decomposition of hardy functions into square integrable wavelets of constant shape," SIAM journal on mathematical analysis, vol. 15, no. 4, pp. 723-736, 1984.

[24] H. Lüders and S. Noachtar, Atlas of Epileptic Seizures and Syndromes. Saunders Company, 2001. 